description can be very complicated. In this case it is worth applying the method of statistical reduction. The main idea of the method of statistical reduction is the following: we replace the real population by a new, reduced population which is distributed according to the same rules as the real population. The process of reduction is a mathematical one based on the theory of probability. As a result of the reduction method we lose information but we gain on simplicity of description. We can lose much information but gain much simplicity and it depends on our choice. In every particular case we are able to regulate this choice in an optimal way. As we know, it is very difficult to compare the structures of two sets with different numbers of points. An additional important advantage of the method of statistical reduction is the fact that different sets after reduction can possess the same number of objects.

As the starting-point in describing the distribution of galaxies we must know the coordinates of galaxies or their numbers in squares by rectangular division of the map. In the first case the method is not only more accurate but also applicable to investigations of individual clusters. Applications to distribution of different extragalactic objects are in progress. In the second case we have already some statistical results. Professor Rudnicki will describe some of them.

\title{
RADIO EMISSION OF ABELL CLUSTERS IN THE GB AND GB2 REGIONS
}

Adam Michalec and Jerzy Machalski

The observational material is based upon pencil-beam surveys made with the NRAO 300-ft radio telescope at $1400 \mathrm{MHz}$. The GB survey (Maslowski 1972) covers $0.1586 \mathrm{sr}$ in the zone $7^{\mathrm{h}} 15^{\mathrm{m}} \leqslant \alpha \leqslant 16^{\mathrm{h}} 20^{\mathrm{m}}$, $459.9 \leqslant \delta<5198$. The GB2 survey made by Machalski in 1975 was carried out in the region defined by $7^{\mathrm{h}} 08^{\mathrm{m}} \leqslant \alpha \leqslant 16^{\mathrm{h}_{5}} 8^{\mathrm{m}}, 31.8 \leqslant \delta \leqslant 39.6$ (0.2828 sr).

The aim of this work is as follows:

(1) to identify the sources in Abe11 clusters,

(2) to estimate the luminosity distribution at $1400 \mathrm{MHz}$ and compare it with that of Owen (1975),

(3) to search for a dependence of spectra on luminosity.

We have obtained the following results:

Number of Abell clusters in the region

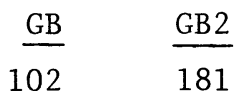

Number of detections within $0.3 \mathrm{r}_{\mathrm{c}}$

(cf. Wills 1966) with $\mathrm{S}_{1400} \geqslant 0.09 \mathrm{Jy}$; with $\mathrm{S}_{1400}<0.09 \mathrm{Jy}$

$\begin{array}{ll}31 * & 39 \\ \text { no data } & 12\end{array}$

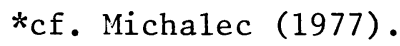


Distances to the clusters were estimated from the relation

$$
\log z_{e}=-4.697+0.224 \mathrm{~m}_{10} \quad \text { (Corwin 1974). }
$$

The luminosity distribution we have obtained is presented in Figure 1 , while Figure 2 shows a spread of spectral indices against luminosity.

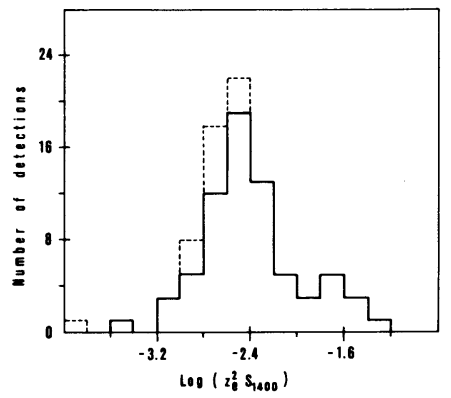

Figure 1. Observed luminosity $\left(z_{\mathrm{e}}^{2} \mathrm{~S}_{1400}\right)$ distribution of GB and $\mathrm{GB} 2$ radio sources stronger than $0.09 \mathrm{Jy}$ which coincide with Abe11 clusters within $0.3 \mathrm{r}_{\mathrm{c}}$ (full line). The dashed line indicates an extension of the distribution to lower flux densities taken from the GB2 survey data.

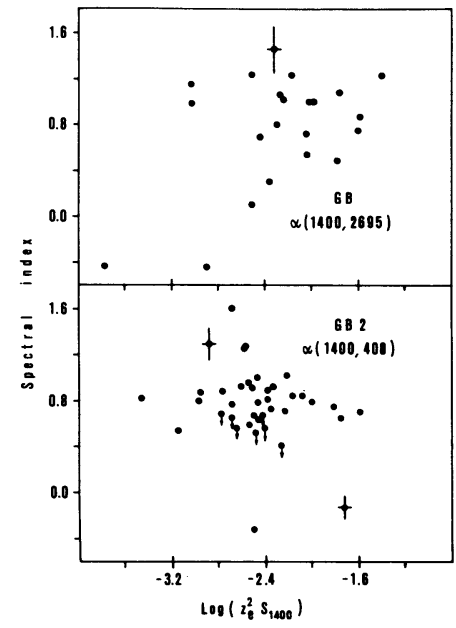

Figure 2. The spread of spectra1index against luminosity for GB sources (upper diagram) and GB2 sources (lower diagram). Vertical and horizontal bars indicate typical rms errors.

Our analysis leads to the following conclusions:

(1) The observed luminosity distribution (based on observations of weaker clusters) is distinctly much more asymmetric in comparison with that given by Owen (1975). This suggests that strong radio galaxies are less numerous in Abell clusters than was estimated by Owen.

(2) There is no evidence for a correlation between spectral index and luminosity (see Figure 2) from the above observations. The spread for the GB data is considerably greater, mainly because of the use of a high frequency spectral index which is more sensitive to cut-offs in this spectral region.

(3) About $10 \%$ of radio sources coinciding with Abell clusters seem to have flat or inverted spectra. For some of them radio structural data are available. These are:

A 0951 (compact radio source, Owen and Rudnick (1976)),

A 1314 (weaker radio source = IC 711; Webber (1974), Vallée and Wilson (1976)). 


\section{REFERENCES}

Corwin, H.G., 1974. Astron. J., 79, 1356. Maslowski, J., 1972. Acta Astron., 22, 227. Michalec, A., 1977. Acta Cosmologica, $6,47$.

Owen, F.N., 1975. Astrophys. J., 195, 593.

Owen, F.N. and Rudnick, L., 1976. Astrophys. J., 203, 307.

Vallée, J.P. and Wilson, A.S., 1976. Nature, 259, 451 .

Webber, J.C., 1974. Publ. Astron. Soc. Pacific, 86, 223. Wi11s, D., 1966. Observatory, 86, 140. 